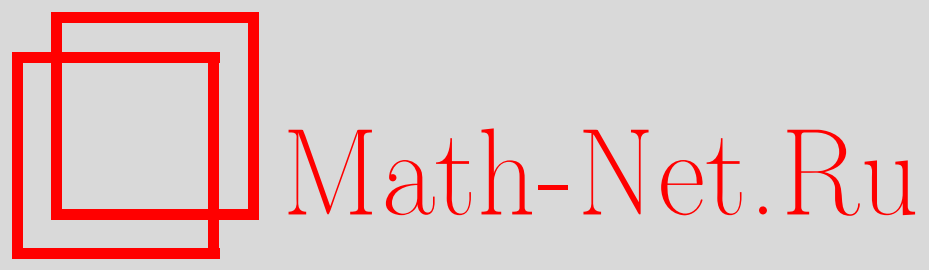

М. Ю. Юркин, О конечномерности задачи о малых колебаниях волчка с полостью, наполненной идеальной жидкостью, Функи. анализ и его прил., 1997, том 31, выпуск 1, 51-66

DOI: https://doi.org/10.4213/faa445

Использование Общероссийского математического портала MathNet.Ru подразумевает, что вы прочитали и согласны с пользовательским соглашением http://www.mathnet.ru/rus/agreement

Параметры загрузки:

IP : 18.208 .226 .222

26 апреля 2023 г., 15:43:56

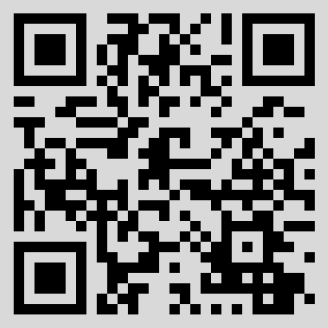


Функииональньй анализ и еәо приложения

1997, т. 31, вып. 1, с. 51-66

УДК 517.9

\title{
О конечномерности задачи о малых колебаниях волчка с полостью, наполненной идеальной жидкостью
}

\author{
(c) 1997. М. Ю. Юркин
}

В настоящей работе исследуется задача о малых колебаниях вращающегося волчка с полостью, целиком заполненной идеальной жидкостью. Предполагается, что полость и оболочка - тела вращения с общей осью симметрии, а точка закрепления оболочки находится на этой оси. Движение происходит в поле силы тяжести. В невозмущенном состоянии жидкость имеет распределение скоростей твердого тела, вращающегося вместе с волчком.

Линейное уравнение малых колебаний волчка с жидкостью в абстрактной форме может быть записано следующим образом:

$$
\dot{u}=M u
$$

где $u$ - вектор некоторого гильбертова пространства $H$, задающий отклонение системы от невозмущенного состояния, а $\boldsymbol{M}$ - ограниченный линейный оператор. В настоящей работе показано, что пространство $H$ может быть разбито на сумму двух подпространств, $H=\boldsymbol{L} \dot{+} \boldsymbol{L}^{\prime}$, таким образом, что оба эти подпространства инвариантны относительно оператора $\boldsymbol{M}$, а все решения уравнения $(*)$ также разлагаются в сумму решений из $\boldsymbol{L}$ и $\boldsymbol{L}^{\prime}$. При этом решениям из $\boldsymbol{L}^{\prime}$ соответствуют движения системы, при которых оболочка волчка равномерно вращается вокруг неподвижной оси симметрии. Обратно, если решению $u(t)$ соответствует движение, при котором ось симметрии волчка неподвижна, то $u(t) \in \boldsymbol{L}^{\prime}$ при любом времени $t$. Иными словами, для того чтобы определить движение оболочки, необходимо и достаточно рассмотреть сужение уравнения $(*)$ на подпространство $\boldsymbol{L}$ (см. п. $9 \S 1)$. Из работы С. Л. Соболева (см. $[1, \S 6])$ следует, что если полость имеет форму эллипсоида, то $\boldsymbol{L}$ конечномерно. А именно если полуоси эллипсоида различны, то $\operatorname{dim} \boldsymbol{L}=6$. Если же полость имеет форму шара, то $\operatorname{dim} \boldsymbol{L}=4$, а влияние жидкости сводится к увеличению моментов инерции и массы волчка.

Назовем задачу о малых колебаниях симметричного волчка с полостью, заполненной жидкостью, относительно состояния равномерного вращения конечномерной, если конечномерно пространство $\boldsymbol{L}$.

Основным результатом настоящей работы является следующая теорема:

Пусть полость есть ограниченная связная область, содержащая точки, лежашие на оси симметрии. Тогда $\boldsymbol{L}$ конечномерно только для полости, имеющей форму слоя между двумя концентрическими подобньми эллипсоидами вращения или эллипсоида вращения (см. теорему 7).

\footnotetext{
^ Работа выполнена при поддержке РФФИ, грант №96-01-00333.
} 
Из нее вытекает, что если полость гомеоморфна шару, то эта конечномерность реализуется только для эллипсоида вращения.

Приводится также ее следствие для волчка с полостью из нескольких связных компонент. О конечности числа зон устойчивости по частоте вращения в этом случае см. замечание 7 (п. $9 \S 2$ ).

Если рассматривать задачу о малых колебаниях жидкости в неподвижном сосуде относительно состояния равномерного вращения, то $\boldsymbol{L}$ конечномерно тогда и только тогда, когда имеется только конечное число собственных колебаний жидкости, обладающих отличным от нуля моментом гидродинамического воздействия на оболочку (см. теорему $2^{\prime}$ и замечание 3 в п. $9 \S 1$ ). Это аналог утверждения, сформулированного во введении к [2].

При выводе уравнений возмущенного движения волчка с осесимметричной полостью С. Л. Соболев пользовался системой координат, равномерно врашающейся вокруг вертикальной оси с угловой скоростью невозмущенного движения волчка. Общее решение уравнений, записанных в этой системе координат, может быть представлено в виде суммы бесконечного числа слагаемых, каждое из которых в свою очередь является решением рассматриваемой задачи. Эти слагаемые различаются между собой тем, какое преобразование они испытывают при повороте волчка на угол $\alpha$. Как показал Соболев, среди этих решений только два соответствуют случаю, когда вместе с жидкостью колеблется и оболочка, причем они являются комплексно-сопряженными, в силу чего достаточно изучать одно из них. Уравнение для этого решения Соболев привел к виду $\dot{R}=i \boldsymbol{B} R$, где $R=(z, w, v), z$ и $w$ - комплексные числа, а $v\left(x_{0}, x_{1}, x_{2}\right)$ - векторное поле в области $\Omega$. Если положить $R^{1}=\boldsymbol{B} R$, где $R^{1}=\left(z^{1}, w^{1}, v^{1}\right)$, то

$$
\begin{gathered}
v_{2}^{1}=-2 \omega i v_{1}+i \frac{\partial p}{\partial x_{2}}+2 \omega^{2} w \frac{\partial \bar{\chi}}{\partial x_{1}}, \quad v_{1}^{1}=2 \omega i v_{2}+i \frac{\partial p}{\partial x_{1}}-2 \omega^{2} w \frac{\partial \bar{\chi}}{\partial x_{2}} \\
v_{0}^{1}=i \frac{\partial p}{\partial x_{0}}, \quad \operatorname{div} v=\operatorname{div} v^{1}=0,\left.\quad v_{n}\right|_{\partial \Omega}=0,\left.\quad v_{n}^{1}\right|_{\partial \Omega}=0 \\
A_{1} w^{1}=A_{2} w+A_{3} z+\int_{\Omega}\left(v_{2} \frac{\partial \chi}{\partial x_{1}}-v_{1} \frac{\partial \chi}{\partial x_{2}}\right) d \Omega, \quad z^{1}=\omega w
\end{gathered}
$$

где $v=\left(v_{0}, v_{1}, v_{2}\right), A_{1}, A_{2}, A_{3}, \omega$ - некоторые константы, а функция $\chi$ определяется из условий

$$
\Delta \chi=0,\left.\quad \frac{\partial \chi}{\partial n}\right|_{\partial \Omega}=x_{0}\left(\cos n x_{2}+i \cos n x_{1}\right)-\left(x_{2}+i x_{1}\right) \cos n x_{0} .
$$

Оператор $\boldsymbol{B}$ самосопряжен в пространстве с индефинитной метрикой, имеющей не более одного отрицательного квадрата, что принципиально для рассматриваемой задачи.

Как и авторы работ $[2,3]$, в настоящей статье мы пользуемся уравнениями движения волчка в системе координат, связанной с твердой оболочкой. При этом, используя симметрию, мы разбиваем решение уравнения $(*)$ в сумму бесконечного числа решений, причем в уравнении $\dot{R}=i \widetilde{\boldsymbol{B}} R$ для того из них, при котором вместе с жидкостью колеблется и оболочка, оператор $\widetilde{\boldsymbol{B}}$ отличен от $\boldsymbol{B}$. Однако из п. $8 \S 1$ следует, что операторы $\widetilde{\boldsymbol{B}}$ и $\boldsymbol{B}$ подобны. 


\section{§1. Уравнения движения волчка и их свойства; определение пространства $L$}

1. Будем рассматривать тело, имеющее полость $\Omega$, целиком заполненную идеальной жидкостью, которое движется вокруг неподвижной точки $O$. Предполагается, что $\Omega$ состоит из конечного числа связных компонент и является телом вращения. Пусть $e_{0}$ - единичный вектор вдоль оси симметрии, а $e_{1}, e_{2}$ таковы, что $O e_{0} e_{1} e_{2}$ - ортонормированный репер, жестко связанный с телом $(O-$ начало координат), и $O x_{0} x_{1} x_{2}$ - координаты в этом репере. Пусть $O f_{0} f_{1} f_{2}$ - ортонормированный репер, жестко связанный с пространством. В невозмущенном состоянии $e_{0}=f_{0}$. Состояние системы описывается тремя величинами: $w \in \mathbb{R}^{3}$ - угловая скорость оболочки, $z \in \mathbb{R}^{3}$ - координаты вектора $f_{0}$ и $v \in J_{0}(\Omega)$ поле скоростей жидкости в системе координат репера $O e_{0} e_{1} e_{2}$. В $\Omega$ справедливо разложение Вейля: $\mathbf{L}_{2}(\Omega)=J_{0}(\Omega) \oplus G(\Omega)$, где $J_{0}(\Omega)$ - замыкание в $\mathbf{L}_{2}(\Omega)$ множества бесконечно дифференцируемых финитных в $\Omega$ соленоидальных векторов, а $G(\Omega)$ состоит из градиентов однозначных в $\Omega$ локально квадратично суммируемых функций с первыми производными, принадлежащими $\mathbf{L}_{2}(\Omega)$ (см. [4]). Определения операторов $\mathrm{div}, \operatorname{rot}, \Delta$ в этих пространствах даются в [4].

2. Пусть $\gamma_{i}, \delta_{i}$ - моменты инерции оболочки и жидкости, рассматриваемой как твердое тело, соответственно относительно $e_{i}, i=0,1,2$. Положим $a_{i}=$ $\gamma_{i}+\delta_{i}, i=0,1,2$. Так как полость и оболочка - тела вращения, то $\gamma_{1}=\gamma_{2}$, $\delta_{1}=\delta_{2}, a_{1}=a_{2}$. Обозначим через $P_{0}$ ортогональный проектор пространства $\mathbf{L}_{2}(\Omega)$ на $J_{0}(\Omega)$. Введем следующие операторы:

$$
\begin{aligned}
B w & =P_{0}([w, x]), & B: \mathbb{C}^{3} \rightarrow J_{0}(\Omega), \\
B^{*} v & =\int_{\Omega}[x, v(x)] d \Omega, & B^{*}: J_{0}(\Omega) \rightarrow \mathbb{C}^{3}, \\
A & =\operatorname{diag}\left\{a_{i}\right\}_{i=0,1,2}, & A: \mathbb{C}^{3} \rightarrow \mathbb{C}^{3},
\end{aligned}
$$

где $x=\left(x_{0}, x_{1}, x_{2}\right)^{T}$, а $[x, y]=\left(x_{1} y_{2}-x_{2} y_{1},-x_{0} y_{2}+x_{2} y_{0}, x_{0} y_{1}-x_{1} y_{0}\right)^{T}$. Без ограничения общности полагаем плотность жидкости равной 1 . Тогда эволюция системы описывается уравнениями

$$
\left(\begin{array}{c}
z \\
A w+B^{*} v \\
B w+v
\end{array}\right)=\left(\begin{array}{c}
{[z, w]} \\
{\left[A w+B^{*} v, w\right]+K\left[z, e_{0}\right]} \\
P_{0}\{[v,(2 w+\operatorname{rot} v)]\}
\end{array}\right)
$$

где $K=g\left(l_{1} M_{1}+l_{2} M_{2}\right)$; здесь $l_{1}$ и $l_{2}$ - расстояния от точки $O$ до центров тяжести, $M_{1}, M_{2}$ - массы оболочки и жидкости соответственно, а $g$ ускорение силы тяжести (см. [3]).

Поясним уравнения системы (1). Первая строка этой системы есть кинематическое соотношение, следующее из определения $z$. Вторая строка - это уравнение, выражающее теорему об изменении кинетического момента системы относительно точки $O$. Третья строка является иной записью уравнений относительного движения жидкости

$$
\dot{v}+(v \nabla) v+2[w, v]=-\nabla p-[\dot{w}, x]-[w,[w, x]], \quad \operatorname{div} v=0 .
$$


Она получается проектированием первого из них на пространство соленоидальных векторных полей, удовлетворяющих условию непротекания на границе области. Подробный вывод этих уравнений имеется в [5].

Невозмущенному движению соответствует следующее стационарное решение системы (1):

$$
u_{0}=\left(z_{0}, w_{0}, v_{0}\right)^{T}, \quad z_{0}=e_{0}, \quad w_{0}=\omega e_{0}, \quad v_{0}=0 .
$$

Подставим в систему (1) решение в виде $u_{0}+u$ и оставим только линейные по $u=(z, w, v)^{T}$ члены. Получим линеаризацию системы (1)

$$
\left(\begin{array}{c}
z \\
A w+B^{*} v \\
B w+v
\end{array}\right)=\left(\begin{array}{c}
{\left[w_{0},(1 / \omega) w-z\right]} \\
{\left[A w_{0}, w\right]+\left[A w, w_{0}\right]+\left[B^{*} v, w_{0}\right]+K\left[z, e_{0}\right]} \\
2 P_{0}\left\{\left[v, w_{0}\right]\right\}
\end{array}\right) .
$$

3. Введем следующие операторы $F, G$ : $F w=\left[e_{0}, w\right], G v=-2 i P_{0}\left\{\left[v, e_{0}\right]\right\}$. Хорошо известно, что $G=G^{*}$ и $\|G\|=2$ (см. [3]). Пусть

$$
J=\left(\begin{array}{ccc}
I & 0 & 0 \\
0 & A & B^{*} \\
0 & B & I
\end{array}\right), \quad M=-i\left(\begin{array}{ccc}
-F & (1 / \omega) F & 0 \\
-(K / \omega) F & \left(a_{0}-a_{1}\right) F & -F B^{*} \\
0 & 0 & i G
\end{array}\right) .
$$

Тогда систему (2) можно записать в виде

$$
J \dot{u}=\omega i M u .
$$

Уравнению (3) соответствует линейный пучок $L(\lambda)=\lambda J-\omega M$.

4. Положим

$$
U_{1}^{s}=\left(\begin{array}{ccc}
1 & 0 & 0 \\
0 & \cos s & -\sin s \\
0 & \sin s & \cos s
\end{array}\right)
$$

В пространстве $J_{0}(\Omega)$ естественным образом действует группа унитарных операторов $U_{2}^{s}$ (напоминаем, что $\Omega$ - тело вращения):

$$
\left(U_{2}^{s} v\right)(x)=U_{1}^{s}\left(v\left(U_{1}^{-s} x\right)\right) .
$$

Таким образом, в пространстве $H=\mathbb{C}^{3} \oplus \mathbb{C}^{3} \oplus J_{0}(\Omega)$ можно определить унитарное семейство $U^{s}=\operatorname{diag}\left\{U_{1}^{s}, U_{1}^{s}, U_{2}^{s}\right\}$. Пусть $T_{k}=(2 \pi)^{-1} \int_{0}^{2 \pi} e^{i k s} U^{s} d s$. Непосредственно из определения вытекают следуюшие свойства: $T_{k}$ - ортогональный проектор при всех $k \in \mathbb{Z}$, причем $\sum_{-\infty}^{+\infty} T_{k}=I$ и $U^{\alpha} T_{k}=e^{-i k \alpha} T_{k}$. Пусть $H_{k}=T_{k} H$; тогда $H=\bigoplus_{-\infty}^{+\infty} H_{k}$, где $T_{k}=\operatorname{diag}\left\{R_{k}, R_{k}, Q_{k}\right\}$, а $R_{k}=0$ при $k \notin\{-1,0,1\}$,

$$
R_{0}=\operatorname{diag}\{1,0,0\}, \quad R_{1}=\frac{1}{2}\left(\begin{array}{ccc}
0 & 0 & 0 \\
0 & 1 & -i \\
0 & i & 1
\end{array}\right), \quad R_{-1}=\bar{R}_{1} .
$$

Справедлива следующая

Лемма 1. $J T_{k}=T_{k} J$ u $M T_{k}=T_{k} M$ nри всех $k \in \mathbb{Z}$. 
ДокАЗАтЕЛЬСтво. Надо показать, что $A U_{1}^{s}=U_{1}^{s} A, F U_{1}^{s}=U_{1}^{s} F, G U_{2}^{s}=$ $U_{2}^{s} G$ и $B^{*} U_{2}^{s}=U_{1}^{s} B^{*}$. Докажем последнее равенство:

$$
\begin{aligned}
B^{*} U_{2}^{s} v & =\int_{\Omega}\left[r, U_{1}^{s} v\left(U_{1}^{-s} r\right)\right] d \Omega=\int_{\Omega}\left[U_{1}^{s} r, U_{1}^{s} v(r)\right] d \Omega \\
& =U_{1}^{s} \int_{\Omega}[r, v(r)] d \Omega=U_{1}^{s} B^{*} v
\end{aligned}
$$

Остальные доказываются аналогично.

СЛЕДСТВИЕ. $J H_{k} \subset H_{k} \quad u M H_{k} \subset H_{k}$ npu всеx $k \in \mathbb{Z}$.

ЗАмЕЧАНИЕ 1 . Непосредственно из определения следует, что $H_{-k}$ состоит из векторов, комплексно-сопряженных к элементам из $H_{k}$.

5. При $k \notin\{-1,0,1\}$ мы имеем

$$
T_{k} J T_{k}=\operatorname{diag}\left\{0,0, Q_{k}\right\}, \quad T_{k} M T_{k}=\operatorname{diag}\left\{0,0, G Q_{k}\right\}
$$

и $H_{k}=\{0\} \oplus\{0\} \oplus Q_{k} J_{0}(\Omega)$, т.е. уравнение $(3)$ при $u \in H_{k}$ описывает движения системы, при которых оболочка равномерно вращается со скоростью $\omega$ вокруг своей неподвижной оси симметрии $e_{0}=f_{0}$. Уравнение движения жидкости в полости совпадает с уравнением малых колебаний жидкости относительно равномерного вращения при неподвижной оболочке.

6. Рассмотрим случай $k=0$. Так $\operatorname{kaK} R_{0}=\operatorname{diag}\{1,0,0\}$, то ему соответствует пространство, к которому относятся те движения системы, при которых $e_{0}=f_{0}$, т.е. направления оси волчка и его угловой скорости неизменны. Если $\gamma_{0}=0$, то говорить о движении оболочки в данном случае не имеет смысла. В случае $\gamma_{0} \neq 0$ нам понадобится следующая

ЛЕмма 2. Пусть $(z, w, v)^{T}$ удовлетворяет уравнению (3) $и \gamma_{0} \neq 0$. Тогда $\left(e_{0}, \dot{w}\right)=0 u\left(e_{0}, B^{*} \dot{v}\right)=0$.

ДокАЗАТЕльство. Умножив обе части уравнения $(3)$ на $\left(0, e_{0}, 0\right)^{T}$, получим $\left(A \dot{w}+B^{*} \dot{v}, e_{0}\right)=0$. Но $B \dot{w}+\dot{v}=\omega i G v$, откуда $\left(B^{*} B \dot{w}, e_{0}\right)+\left(B^{*} \dot{v}, e_{0}\right)=$ $i \omega\left(B^{*} G v, e_{0}\right)=i \omega\left(v, G B e_{0}\right)=0$, поскольку $B e_{0} \in \operatorname{Ker} G$ (см. [3]). Элементарно показывается, что $B^{*} B=\operatorname{diag}\left\{\beta_{i}\right\}_{i=0,1,2}$ (напоминаем, что $\Omega$ - тело вращения). При этом $\beta_{i} \geqslant 0(i=0,1,2)$. Следовательно, $0=\left(\left(A-B^{*} B\right) \dot{w}, e_{0}\right)=$ $\left(a_{0}-\beta_{0}\right)\left(\dot{w}, e_{0}\right)$. Непосредственно из определения вытекает, что $\beta_{0}=\delta_{0}$. Таким образом, так как $\gamma_{0} \neq 0$, то $\left(e_{0}, \dot{w}\right)=0$ и $\left(e_{0}, B^{*} \dot{v}\right)=0$.

Используя лемму 2 и заметив, что $R_{0} F=0$, получаем

$$
T_{0} J T_{0}=\operatorname{diag}\left\{R_{0}, a_{0} R_{0}, Q_{0}\right\}, \quad T_{0} M T_{0}=\operatorname{diag}\left\{0,0, G Q_{0}\right\} .
$$

Таким образом, система уравнений (3) на подпространстве $H_{0}$ описывает движения системы, при которых оболочка врашается вокруг неподвижной оси симметрии $e_{0}=f_{0}$ с постоянной угловой скоростью (возможно, не равной $\omega$ в отличие от предыдущего случая). Уравнение движения жидкости в полости совпадает с уравнением малых колебаний жидкости относительно равномерного вращения при неподвижной оболочке.

7. Рассмотрим случай $k=1$ (случай $k=-1$ разбирается аналогично). В одномерном пространстве $R_{1} \mathbb{C}^{3}$ возьмем в качестве базисного элемента $e=$ $(1 / \sqrt{2})(0,1, i)^{T}$. Тогда $A(\lambda e)=\left(a_{1} \lambda\right) e, F(\lambda e)=\lambda\left[e_{0}, e\right]=-i \lambda e$ и $B(\lambda e)=$ 
$\lambda\left((1 / \sqrt{2}) B e_{1}+(i / \sqrt{2}) B e_{2}\right)=\lambda B e$. Пусть $v \in Q_{1} J_{0}(\Omega)$. В этом случае $\left(B^{*} v, e_{0}\right)$ $=\left(v, B e_{0}\right)=0$, так как $B e_{0} \in Q_{0} J_{0}(\Omega)$,

$$
\left(B^{*} v, e_{2}\right)=\left(B^{*} v, U_{1}^{\pi / 2} e_{1}\right)=\left(U_{1}^{-\pi / 2} B^{*} v, e_{1}\right)=\left(B^{*} U_{2}^{-\pi / 2} v, e_{1}\right)=i\left(B^{*} v, e_{1}\right) .
$$

Таким образом, $B^{*} v=(1 / \sqrt{2})\left(v, B e_{1}+i B e_{2}\right) e=(v, B e) e$. Определим изоморфизм $\Pi: R_{1} \mathbb{C}^{3} \oplus R_{1} \mathbb{C}^{3} \oplus Q_{1} J_{0}(\Omega) \rightarrow \mathbb{C} \oplus \mathbb{C} \oplus Q_{1} J_{0}(\Omega)$ формулой

$$
\Pi(\mu e, \lambda e, v)^{T}=(\mu, \lambda, v)^{T},
$$

т.е. операторам $J, M$ системы (3) будут соответствовать

$$
J_{1}=\Pi J \Pi^{-1}=\left(\begin{array}{ccc}
1 & 0 & 0 \\
0 & a_{1} & b^{*} \\
0 & b & I
\end{array}\right), \quad M_{1}=\Pi M \Pi^{-1}=\left(\begin{array}{ccc}
1 & -1 / \omega & 0 \\
K / \omega & -\left(a_{0}-a_{1}\right) & b^{*} \\
0 & 0 & G
\end{array}\right),
$$

где оператор $b: \mathbb{C} \rightarrow Q_{1} J_{0}(\Omega)$ отождествляется с вектором $b=B e$ (при этом $\left.b^{*} v=(v, b)\right)$. Мы получаем в $\widetilde{H}_{1}=\Pi H_{1}$ задачу

$$
J_{1} \dot{\tilde{u}}=\omega i M_{1} \tilde{u}, \quad \tilde{u}(0)=\tilde{u}_{0},
$$

где $\tilde{u}(t)=(\tilde{z}(t), \widetilde{w}(t), \tilde{v}(t))^{T}$. Соответствуюший пучок $L_{1}(\lambda)$ - это $\lambda J_{1}-\omega M_{1}$. Заметим, что в случае $K=0$ (например, в отсутствие силы тяжести) система (4) распадается на систему

$$
J_{2} \dot{\hat{u}}=\omega i M_{2} \hat{u},
$$

$\hat{u}(t)=(\widetilde{w}(t), \tilde{v}(t))^{T}$ (которой соответствует пучок $\left.L_{2}(\lambda)=\lambda J_{2}-\omega M_{2}\right)$, в пространстве $\mathbb{C} \oplus Q_{1} J_{0}(\Omega)$, где

$$
J_{2}=\left(\begin{array}{cc}
a_{1} & b^{*} \\
b & I
\end{array}\right), \quad M_{2}=\left(\begin{array}{cc}
-\left(a_{0}-a_{1}\right) & b^{*} \\
0 & G
\end{array}\right)
$$

и уравнение $\dot{\tilde{z}}=\tilde{z}-(1 / \omega) \widetilde{w}$.

8. От систем $(4)$ и $\left(4^{\prime}\right)$ перейти к самосопряженным можно различными способами.

Первый способ - это переход к другой системе координат. Пусть

$$
\begin{gathered}
S_{1}=\left(\begin{array}{ccc}
1 /\left(\omega^{2} N\right) & a_{1} /(\omega N) & (1 /(\omega N)) b^{*} \\
1 /(\omega N) & \left(a_{1}+N\right) / N & (1 / N) b^{*} \\
0 & 0 & I
\end{array}\right), \\
S_{2}=\left(\begin{array}{cc}
a_{0} /\left(a_{0}-a_{1}\right) & b^{*} /\left(a_{0}-a_{1}\right) \\
0 & I
\end{array}\right) .
\end{gathered}
$$

где $N=\left(a_{0}-a_{1}\right)-K / \omega^{2}$. Тогда $M_{i} S_{i}$ и $J_{i} S_{i}(i=1,2)-$ самосопряженные операторы, причем $J_{i} S_{i}$ положительны при $N>0$ и имеют одно отрицательное собственное значение при $N<0$.

Второй способ - замена координат и переход к эквивалентной системе урав- 
нений. Положим $S=\left(\begin{array}{ccc}1 & 0 & 0 \\ \omega & 1 & 0 \\ 0 & -b & I\end{array}\right)$. Тогда $S^{-1} J_{1}^{-1} L_{1}(\lambda) S=\lambda I-\omega \tilde{M}$, где

$$
\tilde{M}=\left(\begin{array}{ccc}
0 & -1 / \omega & 0 \\
-\omega N /\left(a_{1}-(b, b)\right) & -c & -b^{*}(G-I) /\left(a_{1}-(b, b)\right) \\
0 & -(G-I) b & G
\end{array}\right)
$$

$c=1-\left(a_{0}-a_{1}+(b,(I-G) b)\right) /\left(a_{1}-(b, b)\right)$. Оператор $\tilde{M}$ является $\tilde{J}$-самосопряженным, где $\tilde{J}=\operatorname{diag}\left\{\omega^{2} N, a_{1}-(b, b), I\right\}$. С точностью до элементарных преобразований оператор $\widetilde{M}$ совпадает с оператором, который исследовал Соболев в [1].

Далее положим $\widetilde{M}_{1}=J_{1}^{-1} M_{1}, \tilde{J}_{1}=S^{-1^{*}} \tilde{J} S^{-1}=\tilde{J}_{1}^{*}$. Легко видеть, что оператор $\widetilde{M}_{1}$ является $\tilde{J}_{1}$-самосопряженным.

9. Будем говорить, что оболочка не участвует в движении системы, соответствующем $u(t)$, если она вращается вокруг неподвижной оси симметрии. Это означает, что $u(t)=\left(z(t), \alpha(t) e_{0}, v(t)\right)^{T}$. В противном случае будем говорить, что оболочка участвует в движении системы.

Пусть $u(t) \in H_{1}$ и оболочка не участвует в движении системы, соответствующем $u(t)$. Тогда $\Pi u(t)=(\tilde{z}(t), 0, \tilde{v}(t))^{T}$.

ОПРЕДЕЛЕНИЕ. Обозначим через $L$ замыкание линейной оболочки системы векторов $\left\{G^{k} b\right\}_{0}^{+\infty}$ в $Q_{1} J_{0}(\Omega)$, и пусть

$$
\begin{gathered}
\tilde{L}^{\prime}=\widetilde{H}_{1} \ominus\left(\operatorname{Lin}\left\{(0,1,0)^{T}\right\} \oplus \operatorname{Lin}\left\{\left(-K / \omega, 0,(G-I) G^{k} b\right)^{T}\right\}_{0}^{+\infty}\right), \\
\tilde{L}=\widetilde{H}_{1} \ominus\left(\tilde{J}_{1} \tilde{L}^{\prime}\right) .
\end{gathered}
$$

Теорема 1. Пусть $\tilde{u}(t)=(\tilde{z}(t), \widetilde{w}(t), \tilde{v}(t))^{T}$ - решение задачи (4). Тогда $w(t) \equiv 0$ в том и только том случае, когда $\tilde{u}(0) \subset \tilde{L}^{\prime}$.

ДокАЗАТЕльСтво. Подставим $\widetilde{w}(t) \equiv 0$ в (4). Получим $\tilde{z}(t)=e^{i \omega t} \tilde{z}(0)$, $\dot{\tilde{v}}=i \omega G \tilde{v},(\dot{\tilde{v}}, b)=i K \tilde{z}(t)+i \omega(\tilde{v}, b)$. Исключая из последнего равенства $\dot{\tilde{v}}$, получаем $i \omega(\tilde{v},(G-I) b)-i K \tilde{z}(t)=0$. Дифференцируя последнее равенство $k$ раз и исключая $\dot{\tilde{v}}$, приходим к равенству $i \omega\left(\tilde{v},(G-I) G^{k} b\right)-i K \tilde{z}(t)=0$. Полагая $t=0$, получаем необходимость условия теоремы. Обратно, пусть $\tilde{u}(0) \subset \tilde{L}^{\prime}$. Решение задачи (4) можно записать в виде $\tilde{u}(t)=\sum_{k=0}^{+\infty}\left(t^{k} / k !\right)(i \omega)^{k} \widetilde{M}_{1}^{k} \tilde{u}(0)$. Но элементарная проверка показывает, что $\tilde{M}_{1} \tilde{L}^{\prime} \subset \tilde{L}^{\prime}$, т. е. $\tilde{u}(t) \subset \tilde{L}^{\prime}$. Это завершает доказательство теоремы.

ТЕОрема 2. Илеет место разложение $\widetilde{H}_{1}=\tilde{L} \dot{+} \tilde{L}^{\prime}$. При этом $\tilde{L}, \tilde{L}^{\prime}$ являются $\widetilde{M}_{1}$-инвариантными и $\tilde{J}_{1}$-ортогональными.

ДокАЗАТЕЛЬСТво. Вторая часть теоремы доказывается непосредственной проверкой (частично она доказана выше). Получим разложение $\widetilde{H}_{1}=\tilde{L} \dot{+} \tilde{L}^{\prime}$. Рассмотрим пространство $\tilde{L} \cap \tilde{L}^{\prime}$. Так как $\tilde{L}$ и $\tilde{L}^{\prime} \tilde{J}_{1}$-ортогональны, а эрмитова форма $\left(\tilde{J}_{1} x, x\right)$ имеет один отрицательный квадрат при $N<0$ и положительна при $N>0$, то $\operatorname{dim} \tilde{L} \cap \tilde{L}^{\prime} \leqslant 1$ (см. [6]). Пусть $x \in \tilde{L} \cap \tilde{L}^{\prime}$. Так как $\tilde{L}, \tilde{L}^{\prime}$ 
$\widetilde{M}_{1}$-инвариантны, то $\widetilde{M}_{1} x=\lambda x$. Поскольку $x \in \tilde{L}^{\prime}$, мы имеем $x=(\alpha, 0, v)^{T}$. Предположим, что $\alpha \neq 0$. Учитывая, что $\left.\tilde{M}_{1}\right|_{\tilde{L}^{\prime}}=\operatorname{diag}\{1,0, G\}$, получаем $\lambda=1$ и $(G-I) v=0$. Вспоминая определение $\tilde{L}^{\prime} \ni x$, получаем $\alpha=0$, т. е. $\alpha$ не может быть отличным от 0 . Но из определения $x$ следует, что $\left(\tilde{J}_{1} x, x\right)=0$. При этом $\left(\tilde{J}_{1} x, x\right)=(v, v)$, т.е. $v=0$ и $x=0$. Аналогично доказывается, что $\left(\tilde{L}+\tilde{L}^{\prime}\right)^{\perp}=0$. Теорема доказана.

ЗАмечАние $2 . \operatorname{dim} \tilde{L}<\infty$ тогда и только тогда, когда $\operatorname{dim} L<\infty$. Действительно, пусть $L$ конечномерно; тогда $\widetilde{H}_{1} \ominus \tilde{L}^{\prime}$ тоже конечномерно. Но $\tilde{L}=$ $\tilde{J}_{1}^{-1}\left(\widetilde{H}_{1} \ominus \tilde{L}^{\prime}\right)$, поскольку $\tilde{J}_{1}=\tilde{J}_{1}^{*}$, т. е. $\tilde{L}$ конечномерно. Обратно, пусть $\tilde{L}$ конечномерно. Обращая приведенное выше рассуждение, получаем, что $\widetilde{H}_{1} \ominus \tilde{L}^{\prime}$ конечномерно. Тогда из определения $\tilde{L}^{\prime}$ следует, что существует такое $n$, что $(G-I) G^{n+1} b=\sum_{k=0}^{n} c_{k}(G-I) G^{k} b$, или $G^{n+2} b=\sum_{k=0}^{n+1} c_{k}^{\prime} G^{k} b$, а это означает конечномерность $L$.

ЗАмЕчАНИЕ 3 . Пусть $\dot{v}=i G v$ и $v(t) \in Q_{1} J_{0}(\Omega)$. Это уравнение описывает малые колебания поля скоростей жидкости в $\Omega$ относительно состояния равномерного вращения (см. [3]). Нетрудно показать, что в этом случае величина $(v,(G-I) b)$ е является моментом сил давления жидкости на оболочку.

ОПРЕДЕЛЕНИЕ. $\boldsymbol{L}=\overline{\Pi^{-1} \tilde{L}} \oplus \Pi^{-1} \tilde{L}, \boldsymbol{M}=i \omega J^{-1} M$,

$$
\boldsymbol{L}^{\prime}=\left(\bigoplus_{k=-2}^{-\infty} H_{k}\right) \oplus \overline{\Pi^{-1} \tilde{L}^{\prime}} \oplus H_{0} \oplus \Pi^{-1} \tilde{L}^{\prime} \oplus\left(\bigoplus_{k=2}^{+\infty} H_{k}\right) .
$$

Суммируя результаты этого пункта, мы можем сформулировать следующую теорему.

Теорема 2'. Пространство $H$ может быть разбито на сумму этих двух подпространств, $\boldsymbol{H}=\boldsymbol{L} \dot{+} \boldsymbol{L}^{\prime}$. Оба эти подпространства инвариантнь относительно оператора $\boldsymbol{M}$, а все решения уравнения (*) разлагаются в сумму решений из $\boldsymbol{L} u \boldsymbol{L}^{\prime}$. При этом решениям из $\boldsymbol{L}^{\prime}$ соответствуют движения системы, при которых оболочка волчка равномерно врачается вокруг неподвижной оси симметрии. Обратно, если решению и $(t)$ coответствует движение, при котором ось симметрии волчка неподвижна, то $u(t) \in \boldsymbol{L}^{\prime}$ при любом времени $t$. Иньми словами, для того чтобы определить движение оболочки, необходимо и достаточно рассмотреть сужение уравнения (*) на подпространство $\boldsymbol{L}$. Подпространство $\boldsymbol{L}$ конечномерно тогда и только тогда, когда конечномерно пространство $L$.

В $\S 2$ мы займемся изучением случаев, когда $\boldsymbol{L}$ конечномерно.

\section{$\S 2$. Теорема о конечномерности пространства $L$ и некоторые ее следствия}

1. Всюду далее (кроме тех мест, где оговорено противное) полагаем, что $\Omega$ - связная ограниченная область. Далее, $h, r, \theta$ - цилиндрические координаты, т. е. $x_{0}=h, x_{1}=r \cos \theta, x_{2}=r \sin \theta$. Как и в $\S 1, e=(1 / \sqrt{2})(0,1, i)^{T} \in \mathbb{C}^{3}$. Напомним, что $b=B e \in Q_{1} J_{0}(\Omega)$ и $\operatorname{rot} b=2 e$. Далее нам понадобится следующая 
ЛЕмма 3. $G^{k} b \in C^{\infty}(\Omega)$ nри всех неотрииательных $k$.

ДокаЗАтельство. Имеем $b=2 i P_{0}\left\{x_{0} e\right\}$, т.е. $b=2 i x_{0} e+\operatorname{grad} p$ и $0=$ $\operatorname{div} b=\Delta p$ в $\Omega$. Таким образом, согласно лемме Вейля (см. [7]), $p \in C^{\infty}(\Omega)$ и $b \in C^{\infty}(\Omega)$. Далее, $G b=-2 i\left[b, e_{0}\right]+\operatorname{grad} p^{\prime}$ и $0=\operatorname{div} G b=-2 i \operatorname{div}\left(\left[b, e_{0}\right]\right)+\Delta p^{\prime}$. Отсюда следует, что так как $b \in C^{\infty}(\Omega)$, то $p^{\prime} \in C^{\infty}(\Omega)$ (лемма Вейля) и $G b \in C^{\infty}(\Omega)$. При остальных $k$ доказательство аналогично.

2. Займемся изучением случая, когда $L$ конечномерно. Пусть $\operatorname{dim} L=n+1$, т. е. $G^{n+1} b \in \operatorname{Lin}\left\{b, G b, \ldots, G^{n} b\right\}$ и $\left\{b, G b, \ldots, G^{n} b\right\}$ - линейно независимая система. Но $G$ самосопряжен, т. е. существуют такие линейно независимые $d_{0}, d_{1}, \ldots, d_{n} \in L$, что $\frac{1}{2} G d_{k}=\lambda_{k} d_{k}, k=0, \ldots, n$ (множитель $1 / 2$ введен для удобства дальнейших вычислений), причем $\lambda_{k} \neq \lambda_{l}(k \neq l)$. Итак, пусть $d \in L$ и

$$
\frac{1}{2} G d=\lambda d \text {. }
$$

Ho

$$
\operatorname{rot} G v=-2 i \frac{\partial}{\partial x_{0}} v
$$

при $v \in J_{0}(\Omega)$ (это доказывается элементарной проверкой). Таким образом,

$$
\frac{\partial}{\partial x_{0}} d=i \lambda \operatorname{rot} d
$$

ЛЕмма 4. Пусть $\operatorname{dim} L=n+1, n>0$. Тогда

$$
\begin{aligned}
& \operatorname{rot}^{n+1} L=0, \\
& \frac{\partial^{n+1}}{\partial x_{0}^{n+1}} L=0 .
\end{aligned}
$$

ДокАЗАТЕЛЬСТво. При $n>0, k=0, \ldots, n$, используя (6), получаем

$$
\frac{1}{(-2 i)^{k}} \operatorname{rot}^{n+1} G^{k} b=\operatorname{rot}^{n+1-k} \frac{\partial^{k}}{\partial x_{0}^{k}} b=\frac{\partial^{k}}{\partial x_{0}^{k}} \operatorname{rot}^{n+1-k} b=0 .
$$

Следовательно, равенство (8) доказано. Используя это равенство, получаем

$$
\partial^{n+1} L / \partial x_{0}^{n+1}=(-2 i)^{n+1} \operatorname{rot}^{n+1} G^{n+1} L=(-2 i)^{n+1} \operatorname{rot}^{n+1} L=0,
$$

т. е. и (9) доказано.

3. В этом пункте мы докажем три леммы.

ОПРЕДЕЛЕНИЕ. $W_{k}$ - это пространства, состоящие из элементов $\varphi\left(x_{1}, x_{2}\right)$, таких, что $\varphi\left(x_{1}, x_{2}\right)=\varphi(r) e^{i k \theta}$ и $\left(0, \partial \varphi / \partial x_{1}, \partial \varphi / \partial x_{2}\right)^{T} \in \mathbf{L}_{2}(\Omega)$.

В дальнейшем $U_{1}^{s}=\left(\begin{array}{cc}\cos s & -\sin s \\ \sin s & \cos s\end{array}\right)$.

Лемма 5. Пусть $\psi \in W_{1} u \Delta \psi=0$ в круге $\{r<R\}$. Тогда $\psi=c r e^{i \theta}$.

ДокАЗАТЕльство. Согласно предыдущему определению, $\psi\left(x_{1}, x_{2}\right)=\psi(r) e^{i \theta}$, т. e.

$$
0=\Delta \psi=\frac{1}{r} \frac{\partial}{\partial r}\left(r \frac{\partial \psi}{\partial r}\right)+\frac{1}{r^{2}} \frac{\partial^{2} \psi}{\partial \theta^{2}}=\left(\frac{1}{r} \frac{\partial}{\partial r}\left(r \frac{\partial \psi(r)}{\partial r}\right)-\frac{1}{r^{2}} \psi(r)\right) e^{i \theta} .
$$


Таким образом, $\psi(r)=c_{1} r+c_{2} / r$, но в нуле $\psi$ ограничена (лемма Вейля), т. е. $c_{2}=0$.

Лемма 6. Пусть $\left(0, \partial \psi / \partial x_{1}, \partial \psi / \partial x_{2}\right)^{T} \in Q_{1} \mathbf{L}_{2}(\Omega)$. Тогда суиествует константа $c$, такая, ито $\psi+c \in W_{1}$.

ДоказАТЕЛЬСТво. Так как $\nabla \psi=U_{1}^{\theta}(\partial \psi(r) / \partial r,(1 / r)(\partial \psi / \partial \theta))^{T}$, то из свойств, указанных в п. $4 \S 1$, получаем

$$
\begin{aligned}
U_{1}^{s}\left(\begin{array}{c}
\partial \psi / \partial x_{1} \\
\partial \psi / \partial x_{2}
\end{array}\right)\left(U_{1}^{-s}\left(\begin{array}{l}
x_{1} \\
x_{2}
\end{array}\right)\right) & =e^{-i s}\left(\begin{array}{c}
\partial \psi / \partial x_{1} \\
\partial \psi / \partial x_{2}
\end{array}\right)\left(\begin{array}{l}
x_{1} \\
x_{2}
\end{array}\right) \\
& =U_{1}^{\theta} e^{-i s}\left(\begin{array}{c}
\partial \psi / \partial r \\
r^{-1} \partial \psi / \partial \theta
\end{array}\right)(r, \theta)
\end{aligned}
$$

С другой стороны,

$$
\begin{aligned}
U_{1}^{s}\left(\begin{array}{c}
\partial \psi / \partial x_{1} \\
\partial \psi / \partial x_{2}
\end{array}\right)\left(U_{1}^{-s}\left(\begin{array}{c}
x_{1} \\
x_{2}
\end{array}\right)\right) & =U_{1}^{s} U_{1}^{\theta-s}\left(\begin{array}{c}
\partial \psi / \partial r \\
r^{-1} \partial \psi / \partial \theta
\end{array}\right)(r, \theta-s) \\
& =U_{1}^{\theta}\left(\begin{array}{c}
\partial \psi / \partial r \\
r^{-1} \partial \psi / \partial \theta
\end{array}\right)(r, \theta-s)
\end{aligned}
$$

Таким образом, полагая $\theta=0, \alpha=-s$, получаем равенство $e^{i \alpha}(\partial \psi / \partial \theta)(r, 0)=$ $(\partial \psi / \partial \theta)(r, \alpha)$ при всех $\alpha$, т.е. $\psi(r, \alpha)=-i e^{i \alpha}(\partial \psi / \partial \theta)(r, 0)+c(r)$. Но $e^{i \alpha}(\partial \psi / \partial r)(r, 0)-(\partial \psi / \partial r)(r, \alpha)=0$. Подставляя сюда предыдущее равенство, убеждаемся, что $0=\left(e^{i \alpha}-1\right) c^{\prime}(r)$. Таким образом, $c(r)=c$. Полагая $\phi=\psi-c$, имеем $\phi(r, \alpha)=-i e^{i \alpha}(\partial \phi / \partial \theta)(r, 0)$, т. е. $\phi(r, \alpha)=e^{i \alpha} \phi(r, 0)$. Лемма доказана.

Лемма 7. Пусть $(0, b, c)^{T} \in Q_{1} \mathbf{L}_{2}(\Omega)$ u $\partial c / \partial x_{1}-\partial b / \partial x_{2}=0$ npu $r^{\prime}<r<r^{\prime \prime}$. Тогда существует такая однозначная функиия $\psi \in W_{1}$, ито $(b, c)^{T}=\nabla \psi$.

ДокАзАтЕльство. Существование неоднозначной функции $\psi-$ известная теорема анализа. Пусть $u=(0, b, c)^{T}$ и $S=\left\{x: r=r_{0}, x_{0}=h_{0}\right\}$. Однозначность $\psi$ следует из того, что

$\int_{S}\left(u, U_{1}^{\theta} e_{2}\right) d \theta=\int_{S}\left(e^{i \theta} U_{1}^{\theta} u\left(h_{0}, r_{0}, 0\right), U_{1}^{\theta} e_{2}\right) d \theta=\int_{S} e^{i \theta} d \theta\left(u\left(h_{0}, r_{0}, 0\right), e_{2}\right)=0$, т. е. циркуляция поля $(0, b, c)^{T}$ вдоль любой окружности $r=r_{0}$ есть нуль. При этом $\psi \in W_{1}$ по лемме 6 .

ОПРЕДЕЛЕНИЕ. $u_{j}(r, \theta)=\left(\prod_{k=1}^{j}\left((2 k+1)^{2}-1\right)^{-1}\right) r^{2 j+1} e^{i \theta}, j=0,1,2, \ldots$.

ЗАМЕЧАНИЕ 4. $\Delta u_{j}=u_{j-1}, \Delta u_{0}=0$.

4. Пусть выполняется (5) и $|\lambda| \neq 1$. Хорошо известно (см. [8, с. 51]), что тогда существует такая функция $P\left(x_{0} ; x_{1}, x_{2}\right)$, что всюду в $\Omega$

$$
\begin{aligned}
& d=\left(\begin{array}{c}
\partial P / \partial x_{0} \\
\frac{\lambda}{\lambda^{2}-1}\left(\begin{array}{cc}
\lambda & -i \\
i & \lambda
\end{array}\right) \nabla P
\end{array}\right), \\
& \Delta P=\frac{1-\lambda^{2}}{\lambda^{2}} \frac{\partial^{2}}{\partial x_{0}^{2}} P,
\end{aligned}
$$

где $\Delta, \nabla$ - двумерные (по переменным $x_{1}, x_{2}$ ) оператор Лапласа и градиент соответственно. 
ЗАмечАниЕ 5. При $d \in J_{0}(\Omega)$ такая функция $P$ из $W_{1}$ единственна, так как 0 - единственная константа в $W_{1}$.

Теорема 3. Пусть $d \in L$ удовлетворяет (5), $|\lambda| \neq 1$ u $\Omega$ содержит точки, лежащие на оси вращения. Тогда существует такой полином $R\left(x_{0}\right)$ $=\sum_{i=0}^{n} M_{n-i} x_{0}^{i}$, что всюду в $\Omega$

$$
P\left(x_{0} ; x_{1}, x_{2}\right)=\sum_{i=0}^{\infty}\left(\frac{1-\lambda^{2}}{\lambda^{2}}\right)^{i} u_{i}\left(x_{1}, x_{2}\right) \frac{\partial^{2 i} R\left(x_{0}\right)}{\partial x_{0}^{2 i}}
$$

ДокАЗАТЕльство. Введем следующие обозначения. Пусть $x \in \Omega$ и

$$
D(x)=\left\{(h, r \cos \theta, r \sin \theta)^{T}: h_{1}<h<h_{2}, r_{1}<r<r_{2}, \theta_{1}<\theta<\theta_{2}\right\} \ni x
$$

- окрестность точки $x$ в $\Omega$. Так как $\Omega$ - тело вращения, то в $\Omega$ содержится и кольцо $\widetilde{D}(x)=\left\{(h, r \cos \theta, r \sin \theta)^{T}: h_{1}<h<h_{2}, r_{1}<r<r_{2}\right\}$. Если $x$ лежит на оси вращения, то под $D(x)$ и $\widetilde{D}(x)$ подразумевается цилиндрическая окрестность вида $\left\{(h, r \cos \theta, r \sin \theta)^{T}: h_{1}<h<h_{2}, 0 \leqslant r<r_{2}\right\}$. Так как $d \in L$, то по лемме 3 имеем $d \in C^{\infty}(\Omega)$ и $P \in C^{\infty}(\Omega)$. По лемме 4 получаем $\partial^{n+1} d / \partial x_{0}^{n+1}=0$ и, следовательно, $\partial^{n+1} P / \partial x_{0}^{n+1}=c=0$ (см. замечание 5$)$. Таким образом, в $D(x)$ имеет место представление

$$
P=\sum_{i=0}^{n} a_{i}\left(x_{1}, x_{2}\right) x_{0}^{n-i}
$$

Используя (11), приходим к равенствам

$$
\Delta a_{j+2}=(n-j)(n-j-1)\left(1-\lambda^{2}\right) \lambda^{-2} a_{j}, \quad \Delta a_{0}=\Delta a_{1}=0
$$

при $r_{1}<r<r_{2}$, причем $a_{j} \in W_{1}, j=0, \ldots, n$. Пусть $y \in \Omega, \widetilde{D}(y) \in \Omega$ и в $\widetilde{D}(y)$ имеет место представление $P=\sum_{i=0}^{n} b_{i}\left(x_{1}, x_{2}\right) x_{0}^{n-i}$. Если $\widetilde{D}=$ $\widetilde{D}(x) \cap \widetilde{D}(y)=\left\{h^{\prime}<h<h^{\prime \prime}, r^{\prime}<r<r^{\prime \prime}\right\}$, то $b_{i}=a_{i}$ при $r^{\prime}<r<r^{\prime \prime}$. Используя лемму 5 , нетрудно показать, что $b_{i}=a_{i}$ при всех $r \neq 0$. Итак, принимая во внимание линейную связность области $\Omega$, получаем, что представление (13), (14) имеет место во всей области. Наличие точек области на оси вращения и лемма 3 приводят к тому, что $a_{j}$ ограничены в нуле. Значит, используя лемму 5 и свойства функций $u_{j}$, мы можем решить систему уравнений $(14)$. После подстановки в (13) получается в точности представление (12).

5. ЛЕмма 8. Пусть $d$ принадлежит $L$ и удовлетворяет (5). Тогда $|\lambda| \neq 1$. ДокАЗАТЕльСтво. Предположим, что $|\lambda|=1$. Из леммы 4 следует, что в любом кольце $\widetilde{D}(x) \in \Omega$ имеет место представление $d=\sum_{i=0}^{n}\left(\begin{array}{l}a_{i}\left(x_{1}, x_{2}\right) \\ b_{i}\left(x_{1}, x_{2}\right) \\ c_{i}\left(x_{1}, x_{2}\right)\end{array}\right) x_{0}^{n-i}$, причем $a_{i}=0$ (см. [3, с. 212]). Подставим это выражение в (7). Тогда мы получим, что $\partial c_{i} / \partial x_{1}-\partial b_{i} / \partial x_{2}=0$. Из $\operatorname{div} d=0$ следует, что $\partial b_{i} / \partial x_{1}+\partial c_{i} / \partial x_{2}=0$. В силу леммы 7 имеем $\left(b_{k}, c_{k}\right)^{T}=\nabla \psi, \Delta \psi=0$. Применяя леммы 5 и 6 , получаем $\left(b_{k}, c_{k}\right)^{T}=M_{k}(1, i)^{T}$, т. е. $d=R\left(x_{0}\right)(0,1, i)^{T}$. Так же как и в теореме 2 , 
показывается, что это представление имеет место во всей области $\Omega$. Очевидно, что это противоречит ограниченности $\Omega$.

Лемма 9. Пусть $d$ принадлежит $L$ и удовлетворяет (5). Тогда $\lambda \neq 0$.

ДокаЗАтЕльство. Пусть $\lambda_{0}=0, G d_{0}=0$ и $d_{0}=\left(d^{0}, d^{1}, d^{2}\right)^{T}$. Тогда $\left(0,-d^{2}, d^{1}\right)^{T}=\left[d_{0}, e_{0}\right]=\left(\partial \psi / \partial x_{0}, \partial \psi / \partial x_{1}, \partial \psi / \partial x_{2}\right)^{T}$. Следовательно, при $x \in \Omega$ в $\widetilde{D}(x)$ имеем $\psi=\psi\left(x_{1}, x_{2}\right), d^{1}=\partial \psi / \partial x_{2}, d^{2}=-\partial \psi / \partial x_{1}$. Кроме того, $\operatorname{div} d_{0}=0$, т.е. $d^{0}=f\left(x_{1}, x_{2}\right)$. При этом $\psi, f \in W_{1}$. Пусть $b=$ $\sum_{k=0}^{n} \alpha_{k} d_{k}$, т. е.

$$
2 e=\operatorname{rot} b=\alpha_{0} \operatorname{rot} d_{0}+\sum_{k=1}^{n} \alpha_{k} \operatorname{rot} d_{k}=\alpha_{0} \operatorname{rot} d_{0}+\sum_{k=1}^{n} \frac{\alpha_{k}}{i \lambda_{k}} \frac{\partial}{\partial x_{0}} d_{k} \text {. }
$$

Но $\operatorname{rot} d_{0}=\left(-\Delta \psi, \partial f / \partial x_{2},-\partial f / \partial x_{1}\right)^{T}$, т.е. $\Delta \psi=\sum_{i=0}^{\infty} N_{i}\left(x_{0}\right) u_{i}\left(x_{1}, x_{2}\right)$ (сумма, очевидно, имеет конечное число ненулевых членов); таким образом, $\psi=\sum_{i=0}^{\infty} K_{i}\left(x_{0}\right) u_{i}\left(x_{1}, x_{2}\right)$. При $\theta=0$ получаем $\psi=\sum_{i=0}^{\infty} L_{i} r^{2 i+1}$ и $d_{0}=$ $\left.\left(\underset{\tilde{D}}{f}, \partial \psi / \partial x_{2},-\partial \psi / \partial x_{1}\right)^{T}\right|_{\theta=0}=(f,(i / r) \psi(r),-\partial \psi(r) / \partial r)^{T}$. Значит, если $\widetilde{D}=$ $\widetilde{D}(x) \cap \widetilde{D}(y) \neq \varnothing$, то в $\widetilde{D}$ имеем $(i / r) \psi_{x}(r)=(i / r) \psi_{y}(r)$ и $L_{x, i}=L_{y, i}$, т.е. $\psi_{x}(r)=\psi_{y}(r)$ при всех $r$. Мы показали, что во всей области

$$
\psi\left(x_{0}, x_{1}, x_{2}\right)=\psi\left(x_{1}, x_{2}\right)=\sum_{i=0}^{\infty} K_{i} u_{i}
$$

(сумма конечна). Аналогично убеждаемся, что $f\left(x_{0}, x_{1}, x_{2}\right)=f\left(x_{1}, x_{2}\right)$ - полином по $r$ при $\theta=0$. Итак, при $\theta=0$ функции $(i / r) \psi(r)$ и $f(r)$ будут полиномами по $r$ и, следовательно, либо являются тождественными нулями, либо имеют конечное число нулей. В случае когда $\psi(r) \equiv 0$, а $f(r) \not \equiv 0$, вектор $(1,0,0)^{T}$ касается $\partial \Omega \cap\left\{x: x_{2}=0\right\}$ во всех точках, что противоречит ограниченности области $\Omega$. Если же $\psi(r)$ не является тождественным нулем, то $\partial \Omega \cap\left\{x: x_{2}=0\right\}$ должна содержать отрезок вертикальной прямой, скажем прямой $x_{1}=r_{0}$. Проверим, выполняется ли это условие для $d_{1}$. Если бы оно выполнялось, то $d_{1}^{1}\left(r_{0}\right)=0$ и, таким образом, $\sum_{i=0}^{\infty} c_{i}\left(r_{0}\right) \partial^{2 i} R_{1}\left(x_{0}\right) / \partial x_{0}^{2 i} \equiv 0$ и $c_{i} \neq 0$. Следовательно, $R_{1}\left(x_{0}\right) \equiv 0, d_{1}=0$, и мы пришли к противоречию.

6. Далее нам понадобится следующая

Лемма 10. Существуют такие числа $\alpha_{k}, k=1, \ldots, n$, ито если $d=$ $\sum_{k=0}^{n} \alpha_{k} d_{k}$, mo $\partial d / \partial x_{0}=\sqrt{2} e$.

ДокАЗАТЕльство. Пусть $b=\sum_{k=0}^{n} \beta_{k} d_{k}$. Тогда непосредственно из определения $L$ следует, что $\beta_{k} \neq 0$. Достаточно положить $\alpha_{k}=\beta_{k} /\left(\sqrt{2} i \lambda_{k}\right)$, что можно сделать в силу леммы 9. Тогда утверждение леммы следует из (7).

7. ЛЕмма 11. Пусть $\Omega$ имеет точки на оси вращения $u G b=\lambda b$, m. e. $\operatorname{dim} L=1$. Тогда

$$
b=\sqrt{2}\left(\begin{array}{c}
i(\lambda-1)\left(x_{1}+i x_{2}\right) \\
i \lambda\left(x_{0}+c_{0}\right) \\
-\lambda\left(x_{0}+c_{0}\right)
\end{array}\right) .
$$

ДокаЗАТЕЛЬСтво. Согласно формуле (7) и условию, $\partial b / \partial x_{0}=2 i \lambda e$. Пусть 
$x$ лежит на оси вращения; тогда в $D(x)$ имеем $b=\left(\begin{array}{c}f_{0}\left(x_{1}, x_{2}\right) \\ \sqrt{2} i \lambda x_{0}+f_{1}\left(x_{1}, x_{2}\right) \\ -\sqrt{2} \lambda x_{0}+f_{2}\left(x_{1}, x_{2}\right)\end{array}\right)$. Условие $\operatorname{div} b=0$ дает нам $\partial f_{1} / \partial x_{1}+\partial f_{2} / \partial x_{2}=0$. Условие $\operatorname{rot} b=2 e$ дает $\partial f_{2} / \partial x_{1}-\partial f_{1} / \partial x_{2}=0, \partial f_{0} / \partial x_{1}=\sqrt{2} i(\lambda-1), \partial f_{0} / \partial x_{2}=\sqrt{2}(1-\lambda)$. Таким образом, согласно лемме $7,\left(f_{1}, f_{2}\right)^{T}=\nabla \psi, \Delta \psi=0, f_{0}=\sqrt{2} i(\lambda-1)\left(x_{1}+i x_{2}\right)$ $+c_{1}$, и в силу лемм 5 и 6 , а также замечания 5 в п. 4 имеем $\psi=c r e^{i \theta}=$ $c\left(x_{1}+i x_{2}\right),\left(f_{1}, f_{2}\right)^{T}=c(1, i)^{T}, c_{1}=0$. Как и в теореме 3, показываем, что это представление имеет место во всей области. Лемма доказана.

Теорема 4. Пусть $\Omega$ имеет точки на оси вращения. Тогда $\operatorname{dim} L=1 в$ том и только том случае, когда $\Omega$ - слой между двумя концентрическими подобньлм эллипсоидами вращения или эллипсоид вращения.

ДокАЗАТЕльство. Необходимость следует из предыдущей леммы, так как осесимметричные поверхности тока для $b$, определенного формулой $(15)$, - это подобные эллипсоиды вращения с полуосями $a, c, c$, отношением квадратов полуосей $a^{2} / c^{2}=(1-\lambda) / \lambda$ и общим центром в точке $\left(-c_{0}, 0,0\right)^{T}$. Достаточность следует из того, что указанная в теореме область односвязна. Таким образом, выполнение равенства (7) для вектора $b$, который, очевидно, совпадает с (15) при соответствующем выборе $\lambda$ и $c$, влечет за собой (5) с $d=b$.

8. ТЕОрема 5. Пусть $\Omega$ имеет точки на оси вращения $u \operatorname{dim} L=n+1$. Тогда $n=0$.

ДОкАЗАТЕЛЬСтво. Пусть $d_{i}, i=0, \ldots, n$, - векторы, определенные в п. 2 , и $n>0$. Мы знаем, что $\lambda_{i} \neq 0,\left|\lambda_{i}\right| \neq 1$ (см. леммы п. 5). Пусть $P_{i}\left(x_{0} ; x_{1}, x_{2}\right), R_{i}\left(x_{0}\right), i=0, \ldots, n$, - введенные в п. 4 функции, соответствующие этим значениям. Так как $d_{i} \in J_{0}(\Omega)$ и $\Omega$ - тело вращения, то очевидно, что $\partial \Omega \cap\left\{x: x_{2}=0\right\}$ будет интегральной линией для полей

$$
g_{i}\left(x_{0}, x_{1}\right)=\left.\left(\begin{array}{c}
\frac{\partial P}{\partial x_{0}}\left(x_{0} ; x_{1}, x_{2}\right) \\
\frac{\lambda_{i}}{\lambda_{i}^{2}-1}\left(\lambda_{i} \frac{\partial P\left(x_{0} ; x_{1}, x_{2}\right)}{\partial x_{1}}-i \frac{\partial P\left(x_{0} ; x_{1}, x_{2}\right)}{\partial x_{2}}\right)
\end{array}\right)\right|_{\left(x_{0}, x_{1}, 0\right)}
$$

- сужений полей $d_{i}$ на плоскость $\left\{x: x_{2}=0\right\}$. Так как $P \in W_{1}$, то $\partial P / \partial x_{2}=$ $(\partial P / \partial r) \sin \theta+i(P / r) \cos \theta$ и на $\left\{x: x_{2}=0\right\}$ имеем $\partial P / \partial x_{2}=\left(i / x_{1}\right) P\left(x_{0} ; x_{1}, 0\right)$. Значит,

$$
g_{i}\left(x_{0}, x_{1}\right)=\left(\begin{array}{c}
\frac{\partial P_{i}}{\partial x_{0}}\left(x_{0} ; x_{1}, 0\right) \\
\frac{\lambda_{i}}{\lambda_{i}^{2}-1}\left(\lambda_{i} \frac{\partial P_{i}\left(x_{0} ; x_{1}, 0\right)}{\partial x_{1}}+\frac{P_{i}\left(x_{0} ; x_{1}, 0\right)}{x_{1}}\right)
\end{array}\right) .
$$

Заметим также, что $u_{j}(r, \theta)=c(2 j) x_{1}^{2 j+1}, j=0,1, \ldots$, при $x_{2}=0$, где $c(2 j)=\prod_{k=1}^{j}\left((2 k+1)^{2}-1\right)^{-1}$. Пусть далее

$$
m_{2 i}(\lambda)=\frac{\lambda}{\lambda^{2}-1}\left(\frac{1-\lambda^{2}}{\lambda^{2}}\right)^{i}((2 i+1) \lambda+1), \quad m_{2 i+1}(\lambda)=\left(\frac{1-\lambda^{2}}{\lambda^{2}}\right)^{i} .
$$


Таким образом, (16) можно записать в виде

$$
g_{k}\left(x_{0}, x_{1}\right)=\left(\begin{array}{c}
x_{1} A_{1}^{k}\left(x_{0}\right)+x_{1}^{3} A_{3}^{k}\left(x_{0}\right)+x_{1}^{5} A_{5}^{k}\left(x_{0}\right)+\cdots \\
A_{0}^{k}\left(x_{0}\right)+x_{1}^{2} A_{2}^{k}\left(x_{0}\right)+x_{1}^{4} A_{4}^{k}\left(x_{0}\right)+\cdots
\end{array}\right),
$$

где

$$
\begin{aligned}
A_{2 i}^{k}\left(x_{0}\right) & =c(2 i) m_{2 i}\left(\lambda_{k}\right) \partial^{2 i} R_{k}\left(x_{0}\right) / \partial x_{0}^{2 i}, \\
A_{2 i+1}^{k}\left(x_{0}\right) & =c(2 i+1) m_{2 i+1}\left(\lambda_{k}\right) \partial^{2 i+1} R_{k}\left(x_{0}\right) / \partial x_{0}^{2 i+1}
\end{aligned}
$$

при $i=0,1, \ldots$, причем $c(2 i+1)=c(2 i)$.

ОПРЕДЕЛЕНИЕ. $\Delta\left(x_{0}\right)=\left(A_{j}^{i}\left(x_{0}\right)\right)_{i, j=0, \ldots, n}$.

ЛЕмма $12 . \operatorname{det} \Delta\left(x_{0}\right) \equiv 0$.

ДокАЗАТЕЛЬСтво. Выберем точку $x_{0}$ так, что $\operatorname{det} \Delta\left(x_{0}\right) \neq 0$ (так как $\operatorname{det} \Delta\left(x_{0}\right)$ - полином, то он может обращаться в нуль лишь в конечном числе точек). Следовательно, векторы $\left.\left(A_{0}^{i}, A_{1}^{i}, \ldots, A_{n}^{i}\right)^{T}\right|_{x_{0}}, i=0, \ldots, n$, образуют базис в $\mathbb{C}^{n+1}$, т. е. при некоторых $\alpha_{k}, k=0, \ldots, n$, имеем $(1,0, \ldots, 0)=$ $\alpha_{0} A^{0}+\cdots+\alpha_{n} A^{n}$. Но это означает, что при этом $x_{0}$ и любом $x_{1}$ имеем $\alpha_{0} g^{0}\left(x_{0}, x_{1}\right)+\cdots+\alpha_{n} g^{n}\left(x_{0}, x_{1}\right)=(0,1)^{T}$. Таким образом, такую линейную комбинацию можно построить для всех $x_{0}$, кроме конечного числа значений, и $\partial \Omega \cap\left\{x: x_{2}=0\right\}$ - интегральная линия для любой линейной комбинации $g_{i}$. Значит, вектор $(0,1)^{T}$ является касательным к границе во всех точках, что противоречит ограниченности области $\Omega$.

Далее полагаем $R_{i}\left(x_{0}\right)=\sum_{k=0}^{n} M_{k}^{i} x_{0}^{n-k}$ и обозначим через $W\left(\lambda_{0}, \ldots, \lambda_{n}\right)=$ $\operatorname{det}\left(\lambda_{j}^{i}\right)_{i, j=0, \ldots, n}$ определитель Вандермонда. Хорошо известно, что если $W\left(\lambda_{0}, \ldots, \lambda_{n}\right)=0$, то существует $i \neq j$, такой, что $\lambda_{i}=\lambda_{j}$.

ЛЕмма 13. $\operatorname{det}\left(m_{i}\left(\lambda_{j}\right)\right)_{i, j=0, \ldots, n}=c W\left(\lambda_{0}, \ldots, \lambda_{n}\right) \prod_{k=0}^{n} \lambda_{k}^{-n}\left(1-\lambda_{k}^{-1}\right)^{-1}$.

ДокАЗАТЕЛЬСТВО. Пусть $\delta=1 / \lambda$; тогда $m_{2 i}(\lambda)=-\left(\delta^{2}-1\right)^{i-1}((2 i+1)+\delta)$ и $m_{2 i+1}(\lambda)=\left(\delta^{2}-1\right)^{i}$. Таким образом, $(1-\delta) m_{l}(\lambda)$ - полином со старшим членом $\delta^{l}$ при $l=0, \ldots, n$, т. е.

$$
\operatorname{det}\left(m_{i}\left(\lambda_{j}\right)\right)_{i, j=0, \ldots, n}=W\left(\delta_{0}, \ldots, \delta_{n}\right) \prod_{k=0}^{n}\left(1-\delta_{k}\right)^{-1} .
$$

Утверждение леммы получается при замене в этом равенстве $\delta_{k}$ на $\lambda_{k}^{-1}$.

СлЕДСтвиЕ. Пусть $\left|\lambda_{k}\right| \neq 1, \lambda_{k} \neq \lambda_{l}($ прu $k \neq l) u \lambda_{k} \neq 0, k=0, \ldots, n$. Tогда $\operatorname{det}\left(m_{i}\left(\lambda_{j}\right)\right)_{i, j=0, \ldots, n} \neq 0$.

ЛЕмма 14. $\prod_{i=0}^{n} M_{0}^{i}=0$.

ДокАЗАТЕльСтво. В силу леммы 12 имеем $\operatorname{det} \Delta\left(x_{0}\right) \equiv 0$. В частности, равен нулю коэфФищиент при старшем члене $x_{0}^{n(n+1) / 2}$ :

$$
\operatorname{det}\left(c(i) \frac{n !}{(n-i) !} m_{i}\left(\lambda_{j}\right) M_{0}^{j}\right)_{i, j=0, \ldots, n}=c \operatorname{det}\left(m_{i}\left(\lambda_{j}\right)\right)_{i, j=0, \ldots, n} \prod_{i=0}^{n} M_{0}^{i} .
$$

Доказательство завершается применением следствия леммы 13.

Итак, согласно лемме 14 , без ограничения общности можно положить $M_{0}^{0}=0$. Обратимся к лемме 10. Полагая $\alpha_{i}$ теми же, что и в ее условии (напомним, что 
это не равные нулю числа), получаем

$$
\left(x_{0}+c, c_{1}, \ldots, c_{n}\right)^{T}=\alpha_{0} A^{0}\left(x_{0}\right)+\cdots+\alpha_{n} A^{n}\left(x_{0}\right) .
$$

Приравняем нулю старшие члены в каждой строке правой части равенства. Получим

$$
\begin{aligned}
& \alpha_{0} c(0) m_{0}\left(\lambda_{0}\right) M_{0}^{0}+\alpha_{1} c(0) m_{0}\left(\lambda_{1}\right) M_{0}^{1}+\cdots+\alpha_{n} c(0) m_{0}\left(\lambda_{n}\right) M_{0}^{n}=0, \\
& \alpha_{0} c(1) m_{1}\left(\lambda_{0}\right) M_{0}^{0}+\alpha_{1} c(1) m_{1}\left(\lambda_{1}\right) M_{0}^{1}+\cdots+\alpha_{n} c(1) m_{1}\left(\lambda_{n}\right) M_{0}^{n}=0, \\
& \ldots \ldots \ldots \ldots \ldots \ldots \ldots \ldots \ldots \ldots \ldots \ldots \ldots \ldots \ldots \ldots \ldots \ldots \ldots \ldots \ldots \ldots \ldots \ldots \ldots \ldots \ldots \ldots \ldots \ldots \ldots \ldots \\
& \alpha_{0} c(n) m_{n}\left(\lambda_{0}\right) M_{0}^{0}+\alpha_{1} c(n) m_{n}\left(\lambda_{1}\right) M_{0}^{1}+\cdots \cdots+\alpha_{n} c(n) m_{n}\left(\lambda_{n}\right) M_{0}^{n}=0 .
\end{aligned}
$$

Учитывая равенство $M_{0}^{0}=0$ и следствие леммы 13 , примененное к определителю $\operatorname{det}\left(m_{i}\left(\lambda_{j}\right)\right)_{i, j=0, \ldots, n}$, получаем, что $M_{0}^{i}=0, i=0, \ldots, n$. Поэтому мы можем применить лемму 14 к системе $\left\{d_{0}, \ldots, d_{n-1}\right\}$ и получить, что $\prod_{i=0}^{n-1} M_{1}^{i}$ $=0$. Без ограничения общности полагаем $M_{1}^{0}=0$. Применив лемму 14 к системе $\left\{d_{1}, \ldots, d_{n}\right\}$, получим, что $\prod_{i=1}^{n} M_{1}^{i}=0$. Без ограничения общности полагаем $M_{1}^{1}=0$. Запишем систему уравнений, получаемую из (18) приравниванием нулю старших членов. Действуя аналогично предыдущему шагу, получаем $M_{1}^{i}=0$, $i=0, \ldots, n$. Совершенно аналогично доказывается, что $M_{k}^{i}=0, i=0, \ldots, n$, $k=2, \ldots, n-2$. Наконец, применяя лемму 14 к парам векторов $d_{i}$, получаем, что $M_{n-1}^{i}=0, i=0, \ldots, n-1$. Значит, если $n>0$, то $R_{0}\left(x_{0}\right) \equiv c$ при некоторой нумерации полиномов $R_{i}$. Следовательно, $d_{0}=c(0,1, i)^{T}$, что противоречит ограниченности области $\Omega$. Теорема 5 доказана.

Итак, объединяя теоремы 5 и 4, получаем такой результат:

Теорема 6. Пусть $\Omega$ имеет точки на оси вращения $u \operatorname{dim} L=m$. Тогда $m=1$ и $\Omega$ - слой между двумя концентрическими подобньми эллипсоидами вращения или эллипсоид вращения.

ТЕОРЕМА 7. Пусть полость есть ограниченная связная область, содержашая точки, лежащие на оси симметрии. Тогда $\boldsymbol{L}$ конечномерно только для полости, имеюшей форму слоя между двумя кониентрическими подобньлм эляипсоидами вращения или эллипсоида вращения.

СЛЕдСТВИЕ. Если полость гомеоморфна шару, то эта конечномерность реализуется только для эллипсоида вращения.

Доказательство теоремы 7 вытекает из теорем 6 и 2'. Указанное следствие элементарно, так как односвязность влечет за собой наличие точек области на оси вращения.

ЗАмЕчАниЕ 6. Можно показать, что утверждения п. 4 работы [9] означают, что $G b=\lambda b$ в случае, когда $\Omega$ - область, ограниченная софокусными эллипсоидами вращения; таким образом, $\operatorname{dim} L=1$. Теорема 4 показывает, что это утверждение неверно. Более того, из теоремы 6 следует, что в этом случае $\operatorname{dim} L=\infty$. 
9. Однако если отказаться от требования связности области $\Omega$, то можно реализовать случай $\operatorname{dim} L=m$ при любом $m>1$. Об этом говорит следующая

Теорема 8. Пусть $\Omega=\bigcup_{i=0}^{n} \Omega_{i}$, где $\Omega_{i}$ - непересекаюшиеся свлзные области, каждая из которых есть слой между двумя кончентрическими подобными эллипсоидами врачения, причем если $a_{i}, c_{i}, c_{i}$ обозначают полуоси внешнего әллипсоида для $\Omega_{i}$, то $a_{i}^{2} / c_{i}^{2}$ различны. Тогда $\operatorname{dim} L=n+1$.

ДокАЗАТЕЛЬСТВо. Если $\Omega$ состоит из нескольких связных компонент, то пространство $J_{0}(\Omega)$ естественным образом разбивается в прямую сумму: $J_{0}(\Omega)$ $=\bigoplus_{i=0}^{n} J_{0}\left(\Omega_{i}\right)$. При этом оператор $G$ и проекторы $Q_{k}$, очевидно, коммутируют с ортогональными проекторами $S_{i}$ на подпространства $J_{0}\left(\Omega_{i}\right)$. Доказательство теоремы завершает тот факт, что $d_{i}=S_{i} b$ являются собственными векторами оператора $G$ с собственными значениями $\lambda_{i}=c_{i}^{2} /\left(a_{i}^{2}+c_{i}^{2}\right)$ соответственно.

ЗАмечание 7 . Рассмотрим $\Omega$ из условия теоремы 8 . Под устойчивостью задачи (3) будем понимать вещественность спектра пучка $L(\lambda)$. Так как спектр оператора $G$ вещественный (см. [3]), $\left.\widetilde{M}_{1}\right|_{L^{\prime}}=\operatorname{diag}\{1,0, G\}$ и $L$ конечномерно, то устойчивость имеет место, если $\left.\operatorname{det} L_{1}(\lambda)\right|_{\tilde{L}}$ обращается в 0 только при вещественных $\lambda$. Рассуждения, аналогичные приведенным в [1], показывают, что при изменении параметра $\omega$ имеется конечное число промежутков вещественной оси, где теряется устойчивость.

Автор искренне благодарен А. Г. Костюченко за постановку задач и постоянное внимание к работе.

\section{ЛитеРАтУРА}

1. Соболев С. Л. О движении симметричного волчка с полостью, наполненной жидкостью. ПМТФ, №3, 20-55 (1960).

2. Иилинский А. Ю., Темченко М. Е. О малых колебаниях вертикальной оси волчка, имеющего полость, целиком наполненную идеальной несжимаемой жидкостью. ПМТФ, №3, 65-75 (1960).

3. Копачевский Н. Д., Крейн С. Г., Нго Зуй Кан. Операторные методы в линейной гидродинамике: Эволюционные спектральные задачи. Наука, М., 1989.

4. Ладыженскал O. А. Математические вопросы динамики вязкой несжимаемой жидкости. Наука, М., 1970.

5. Моисеев Н. Н., Румянцев В. В. Динамика тела с полостями, содержащими жидкость. Наука, М., 1965.

6. Понтрягин Л. С. Эрмитовы операторы в пространстве с индефинитной метрикой. Изв. АН СССР, сер. матем., 8, №6, 243-280 (1944).

7. Рид М., Саймон Б. Методы современной математической физики, т. 2. Мир, M., 1977.

8. Гринспен $X$. Теория вращающихся жидкостей. Гидрометеоиздат, Л., 1975.

9. Рвалов Р. В., Роговой В. М. О вращательных движениях тела с полостью, содержащей жидкость. Механика твердого тела, №3, 15-20 (1972).

Московский государственный университет, механико-математический факультет
Поступило в редакцию 31 октября 1995 г. 